\title{
Pautas territoriales de la fecundidad en la Región Metropolitana de Barcelona (1986-2010)
}

\section{Spatial patterns of fertility in the Barcelona Metropolitan Region (1986-2010)}

\author{
Isabel Pujadas Rúbies, Jordi Bayona-i-Carrasco, Fernando Gil-Alonso \\ y Cristina López Villanueva*
}

\section{INTRODUCCIÓN}

Dos procesos muy intensos han determinado la (re) distribución de la población de las regiones metropolitanas españolas durante las últimas décadas: la suburbanización o redistribución interna de la población por movilidad residencial, mayoritariamente desde los centros metropolitanos hacia sus periferias (Módenes, 1998; Pujadas et al., 2012; Feria y Susino, 2012); y la incorporación de un elevado número de residentes extranjeros en el contexto del «boom» inmigratorio experimentado en nuestro país (Bayona et al., 2011; Pozo y García Palomares, 2011). Ambos procesos han seleccionado la población y han contribuido a la transformación de unas metrópolis cada vez más

\footnotetext{
* Este trabajo forma parte del proyecto de I+D "Las nuevas ciudades españolas. El impacto espacial de las dinámicas demográficas recientes en las grandes áreas urbanas españolas en un contexto de crisis" (CSO2011-24680), dirigido por la Dra. Isabel Pujadas. Los investigadores forman parte del grupo de investigación consolidado "Territori, Població i Ciudadania", reconocido por la Generalitat de Cataluña (2009SGR01086) y dirigido por la Dra. Isabel Pujadas. Una primera versión de este trabajo se presentó en el XIII Congreso de la Población Española, en septiembre de 2012 en Santander.

Departamento de Geografía Humana, Universitat de Barcelona (ipujadas@ub.edu; jordibayona@ub.edu; fgil@ub.edu; clopez@ub.edu).
} 
fragmentadas y menos homogéneas, en comparación a lo que sucedía hace un cuarto de siglo. A estos procesos hay que añadir las transformaciones derivadas de la Segunda Transición Demográfica (Lesthaege, 1995 y Van de Kaa, 1987), a las cuales se ha prestado escasa atención desde los estudios urbanos (Reques, 2011), a pesar de constituir un elemento importante de transformación espacial (Buzar et al., 2005). El aumento del número de hogares y la reducción del tamaño medio de los mismos ha discurrido de forma paralela al incremento de las rupturas matrimoniales y de pareja, a la reconstitución de familias y al aumento de la cohabitación, fenómenos que, junto al descenso y el retraso de la fecundidad, no se han producido con la misma intensidad y calendario en todo el territorio metropolitano (Pujadas y López-Villanueva, 2005), siendo pioneras las ciudades centrales en los nuevos comportamientos, que después se difunden al resto del área metropolitana.

En efecto, la creciente diferenciación en el espacio metropolitano se manifiesta, también, en los comportamientos demográficos, siendo la fecundidad uno de los más destacados. A pesar de la importancia que se le da a los aspectos territoriales, especialmente del contexto urbano (tipología del vecindario y distancia del centro; características de las viviendas predominantes en cada barrio, incluyendo superficie, calidad y precio promedios; servicios públicos disponibles; sistemas de transporte, etc.) en las decisiones sobre la fecundidad - $\mathrm{o}$ en las decisiones sobre cambios de domicilio ligadas a una ampliación del tamaño de la familia - existe, como indica Boyle (2003) poco interés desde la geografía de la población en el estudio reciente de dicho fenómeno. En Europa, pocos estudios abordan este tema. Si bien es cierto que existen trabajos como los de Courgeau (1989) en los que se relaciona la movilidad residencial con la formación familiar, u otros como Michielin (2004) en los que se analiza la baja fecundidad en los contextos urbanos y el papel de la migración en este proceso, es en los trabajos de Kulu y Boyle (2009) o de Kulu et al. (2007 y 2009) donde se aborda esta perspectiva al comparar, para los países nórdicos, los valores de la fecundidad metropolitana y su evolución. Los resultados obtenidos indican una mayor fecundidad suburbana, una maternidad a edades más avanzadas en los centros metropolitanos, y la persistencia de dichas diferencias después de controlar por las características sociodemográficas de las madres. Una migración suburbana selectiva de parejas, pero también una mayor fecundidad de los residentes en áreas no centrales, explicarían los resultados obtenidos.

En el caso de España, las aportaciones más destacadas sobre el análisis territorial de la fecundidad metropolitana corresponde a los trabajos de Pozo y Rodríguez Moya (2002 y 2003) para la Comunidad Autónoma de Madrid, y el 
de Pujadas y López-Villanueva (2005) para la Región Metropolitana de Barcelona. Un mayor detalle municipal y la actualización de los resultados, cuestión especialmente importante después del boom migratorio internacional, son las principales aportaciones del artículo en relación a la bibliografía precedente. Los resultados de dichos trabajos apuntan en la misma dirección: valores significativamente diferentes en la intensidad y el calendario de la fecundidad dentro de las respectivas áreas metropolitanas, con un centro menos fecundo y con un calendario más tardío, al tiempo que las periferias muestran una mayor fecundidad en el periodo analizado. La localización en la periferia de las parejas jóvenes con proyectos reproductivos explicaría esta fecundidad diferencial (Pujadas, 1992; Cabré y Módenes, 1997).

Así pues, partimos en esta investigación con la hipótesis de que, mientras que en España las diferencias provinciales en la intensidad de la fecundidad tienden progresivamente a reducirse, las desigualdades en los niveles de fecundidad dentro de una misma área metropolitana estarían, por el contrario, aumentando y diversificándose según la tipología del municipio (urbe central, municipio de gran tamaño de la primera corona o continuo urbano, localidades suburbanas de tamaño medio o pequeño...). El objetivo de este trabajo es estudiar las diferencias de intensidad de la fecundidad en el interior de la Región Metropolitana de Barcelona (RMB), que se toma como caso de estudio, para el periodo 1986-2010. Se trata de un ámbito fuertemente urbanizado en el que se han asentado alrededor de 750 mil personas de nacionalidad extranjera en la primera década del siglo xxI, un periodo en el que la movilidad interna (cambios de municipio de residencia dentro de la RMB) aumentaba progresivamente hasta alcanzar un máximo de cerca de 160 mil migraciones intra-metropolitanas anuales en el año 2006, para descender ligeramente a continuación como consecuencia de la crisis económica e inmobiliaria, hasta los 146 mil movimientos de 2010.

El fuerte impacto de la migración internacional en la RMB podría poner en cuestión la validez de la hipótesis de partida. En efecto, el análisis de la fecundidad de las mujeres extranjeras indica para España unos niveles de fecundidad más elevados —especialmente en el caso de las africanas-, aunque con una fecundidad descendiente en el tiempo. Devolder y Cabré (2009) analizan el impacto de dicha fecundidad sobre el crecimiento del ISF total en España, con el resultado de que el crecimiento reciente del Índice Sintético de Fecundidad (ISF) general se debe, en su mayor parte, a la contribución de la población extranjera. Aparecen, además, importantes diferencias en el calendario de la fecundidad, mucho más rejuvenecido que el de las autóctonas (Castro y Rosero-Bixby, 2011). Estos mismos patrones se repiten en Cataluña (Devolder 
et al., 2011). Dado que los inmigrantes tienen tendencia a concentrarse, al menos en una primera fase, en los ámbitos centrales de las metrópolis (Bayona y Gil-Alonso, 2012) ello significaría que su asentamiento podría haber hecho posible un aumento y rejuvenecimiento de la fecundidad de las ciudades centrales. Sin embargo, el posterior desplazamiento de los migrantes extranjeros — sobre todo a medida que se asientan de un modo más estable en nuestro país - hacia municipios metropolitanos, significaría que una parte quizás mayoritaria-, de su fecundidad se produciría en estos últimos municipios y no en la ciudad central. Por lo tanto, la presencia significativa de población extranjera en la RMB no negaría la validez, al menos a priori, de la hipótesis inicial.

\section{DATOS UTILIZADOS Y CARACTERÍSTICAS DEL ÁREA DE ANÁLISIS}

Para el análisis de la fecundidad se han utilizado los datos del Movimiento Natural de la Población (MNP) publicados por el Institut d'Estadística de Catalunya (Idescat) correspondientes a los nacimientos por edad de la madre y lugar de residencia de ésta, y a la serie de población "estimada», es decir, residente en cada unidad territorial según estimaciones efectuadas por el Idescat. En concreto, se han tomado las estimaciones intercensales de población entre 1986 y 2001, y las estimaciones postcensales desde 2001 a 2010. Hemos preferido utilizar estas series de población estimada como stock de referencia utilizado en el denominador para el cálculo de las tasas específicas de fecundidad, en vez de utilizar la población oficial proporcionada por el Padrón continuo (INE), porque pensamos que éste último sobreestima la población extranjera (Gil-Alonso, 2010) y, por lo tanto, la población de las unidades geográficas analizadas. Como consecuencia, ello habría dado lugar a unos índices de fecundidad inferiores, lo que habría creado distorsiones que serían de especial importancia en un fenómeno como el aquí analizado, cuyos valores se encuentran marcados por una baja intensidad ${ }^{1}$. Por el contrario, se considera que la población estimada por Idescat se aproxima más a la población real, aunque esta elección no está exenta de problemas, pues si bien se dispone de los nacimientos por detalle municipal, en cambio la población es-

\footnotetext{
${ }^{1}$ Por ejemplo, y para la provincia de Barcelona, el ISF calculado para el año 2010 y con datos del padrón continuo sería de 1,40 hijos por mujer, mientras que la utilización de poblaciones estimadas como población de referencia produciría un indicador de 1,51 hijos por mujer. Esta última cifra es la que el INE publica como valor de fecundidad de la provincia catalana.
} 
timada únicamente se ha publicado para los municipios mayores de $45 \mathrm{mil}$ habitantes, así como también a nivel comarcal, lo que ha permitido calcular asimismo las tasas correspondientes a los «restos comarcales» (población estimada de la comarca menos la que reside en municipios mayores de 45 mil habitantes). Una limitación importante de esta decisión es la imposibilidad de profundizar en la fecundidad diferencial según la nacionalidad, ya que no se dispone en la población estimada de los datos desagregados según esta variable. En cambio, un argumento adicional a favor de la utilización de las estimaciones de población es que los resultados así obtenidos a escala municipal son comparables con los cálculos de fecundidad que proporcionan los institutos de estadística oficiales, ya sea el Instituto Nacional de Estadística (INE) o el Idescat, que de forma análoga utilizan estimaciones de población para el cálculo de los indicadores de fecundidad ${ }^{2}$.

Por lo tanto, los indicadores de fecundidad se han calculado anualmente desde 1986 hasta 2010, a partir de los datos del Idescat para los 19 municipios

\section{FIGURA 1}

\section{DISTRIBUCIÓN DE LOS MUNICIPIOS DE MÁS DE 45 MIL HABITANTES Y RESTOS COMARCALES}

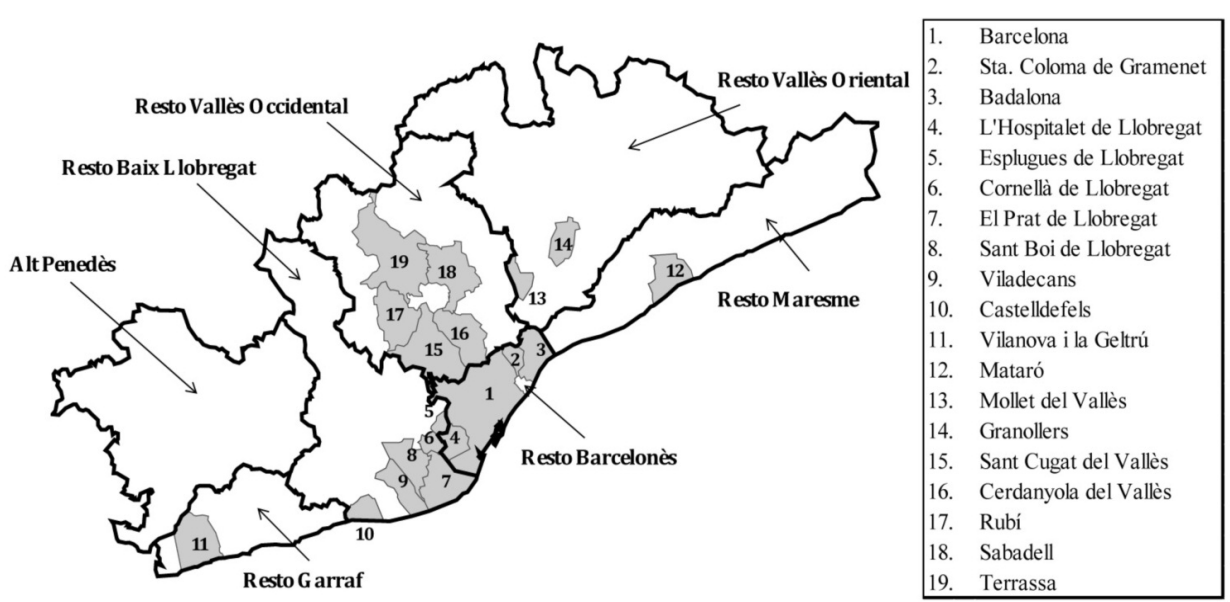

Fuente: elaboración propia.

${ }^{2}$ En el caso del INE, la población de referencia estimada proviene de las EPOBAs (Estimaciones de Población Actual). 
mayores de 45 mil habitantes de la RMB y para los 7 restos comarcales, con el resultado de poder subdividir la Región Metropolitana de Barcelona en 26 unidades de análisis (figura 1). Se ha utilizado la definición clásica de la RMB o Ámbito Metropolitano compuesta por 164 municipios de siete comarcas que, en conjunto, tenían $3.236 \mathrm{~km}^{2}$ y 5.029.181 habitantes a 1 de enero de 2011 .

\section{DiNÁMICAS METROPOLITANAS RECIENTES: SUBURBANIZACIÓN E INMIGRACIÓN INTERNACIONAL}

La Región Metropolitana de Barcelona conoce, durante las últimas décadas, un intenso proceso de descentralización, con una movilidad residencial creciente entre un centro emisor - formado por la ciudad de Barcelona y los municipios del continuo urbano o primera corona metropolitana- y una periferia receptora de migrantes internos, donde los municipios menores y más alejados de la ciudad central son los que obtienen los saldos migratorios positivos más elevados en valores relativos (Pujadas, 2009; Alberich, 2010; Bayona y Pujadas, 2014). Esta dinámica redistribuye la población y especializa el territorio, puesto que los movimientos migratorios son de naturaleza selectiva. A este proceso se suma, de forma reciente, la llegada de población extranjera, que durante los últimos años participan activamente de los procesos de movilidad residencial, aunque se dirigen, a diferencia de los españoles, hacia las ciudades de la primera corona metropolitana (Bayona y Gil-Alonso, 2012).

La ciudad de Barcelona alcanzó su máximo de población en el año 1981 con 1.752.627 habitantes; a partir de esta fecha su población se redujo hasta alcanzar un mínimo por debajo del millón y medio de personas en el año 2000, para empezar a recuperar población desde ese año gracias a la llegada de inmigrantes internacionales, con un nuevo máximo de 1.621.537 en 2009. En ese año, el 17,5\% de su población era de nacionalidad extranjera. Desde entonces, y como consecuencia de la ralentización de los flujos migratorios asociada a la crisis económica, la ciudad vuelve a perder habitantes de nuevo, situándose en 2011 en 1.615.448 habitantes, con un 17,2\% de extranjeros (porcentaje que aumenta hasta el 21,2\% si tomamos la población nacida en el extranjero, cifra que a pesar del descenso de extranjeros —en parte por adopción de la nacionalidad española- sigue aumentando).

En el conjunto de la RMB, el periodo 1981-1996 también se caracterizó por el estancamiento de su población. En esta etapa se observa un ligero decrecimiento de 10 mil residentes, de los 4.238 .876 de 1981 a los 4.228 .048 de 1996. Es a partir de entonces, con la masiva incorporación de población ex- 


\section{FIGURA 2}

\section{SALDO MIGRATORIO NETO, 2000-2009, Y PROPORCIÓN DE RESIDENTES EXTRANJEROS EN LA RMB (2010)}

Saldo migratorio neto intra-metropolitano

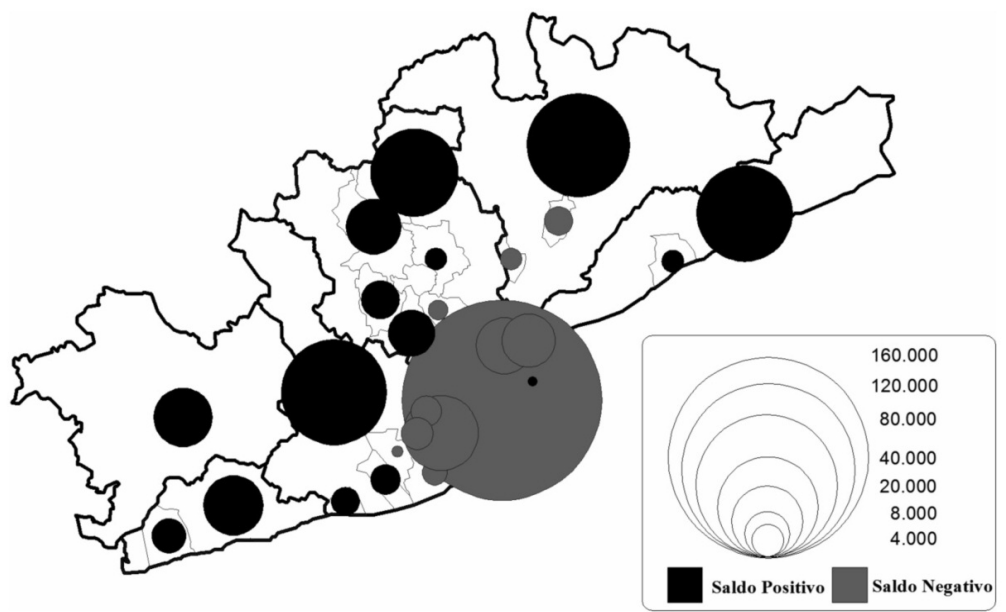

Proporción de extranjeros

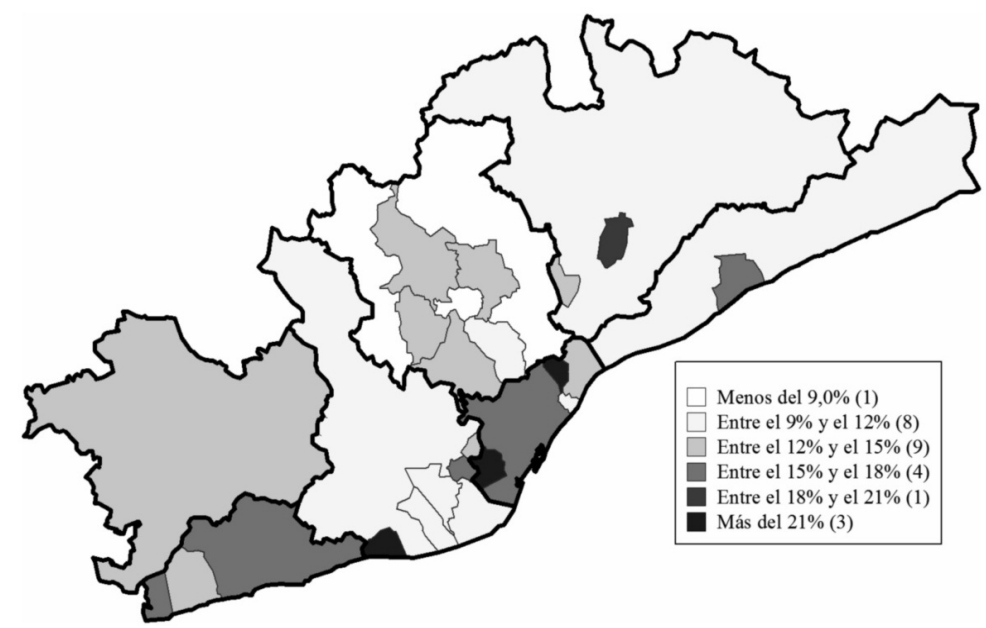

Fuente: elaboración propia a partir del Padrón de Población (2010) y la Estadística de Variación Residencial (2000-2009), con datos del INE. 
tranjera, cuando se abre una nueva época de crecimiento, hasta alcanzar los 5.012.961 habitantes de 2010. En este contexto, el peso de la ciudad central en el conjunto de la RMB disminuye constantemente, del 41,3\% que representaba en 1981 al 32,3\% de 2010.

También el impacto de la inmigración internacional se ha extendido al conjunto de la RMB, pues los inmigrantes extranjeros no sólo son muy numerosos (733.769 extranjeros y el 14,6\% de la población de la RMB en 2011) sino que se encuentran presentes en todo el territorio metropolitano. Nacionalidades que fueron primero importantes en Barcelona se difunden con posterioridad por el territorio metropolitano, donde además se observa que tienen estructuras de hogares con mayor presencia de núcleos familiares ${ }^{3}$ que aquéllas asentadas en la urbe central, donde el peso de hogares unipersonales y sin núcleo familiar es mayor. Ello se podría interpretar en el sentido de que a medida que los inmigrantes extranjeros se asientan de una manera más estable en el territorio y consolidan sus formas familiares, tienen tendencia, al igual que los autóctonos, a salir de la ciudad central (Domingo y Bayona, 2007). Para muchos de ellos la ciudad central ha funcionado como espacio de primera incorporación y asentamiento, sumándose progresivamente a los flujos de suburbanización del conjunto de la población — los saldos negativos de Barcelona y los municipios más próximos se observan en la tabla 1 y la figura $2-$ y se han ido estableciendo en las periferias metropolitanas, donde aumentan su peso en la población total (figura 2, abajo), con una particular preferencia por los municipios de la primera corona o continuo urbano, como es el caso de LHospitalet de Llobregat (donde constituyen el 22,6\% de la población), Santa Coloma de Gramenet (el 22,5\%) o Badalona (el 14,8\%), por citar sólo algunos de los más significativos (Bayona y Gil-Alonso, 2012).

El análisis de la distribución por nacionalidad de la población extranjera nos indica la existencia de diferencias importantes en la composición por nacionalidades del stock de residentes extranjeros a escala metropolitana. En la ciudad de Barcelona, en los municipios de su contiguo urbano y en otros que concentran población de clase media-alta hay poca presencia de africanos, que sólo representan el 7,3\% de los extranjeros en Barcelona, un 6,1\% en Sant Cugat del

${ }^{3}$ Utilizando la definición de núcleo familiar propuesta por Peter Laslett (1972), y a partir de los datos del Censo de 2001, los residentes extranjeros de los municipios de la primera corona metropolitana residían en mayor medida en hogares formados por parejas con o sin hijos (un 56,1\%) que en Barcelona ciudad (un 47,7\%). Al contrario, las estructuras menos familiares (hogares unipersonales y sin núcleo familiar) se encontraban más representadas en el centro metropolitano, situaciones que se repetían en el análisis por nacionalidad. 
Vallès, o un 3,2\% en Sitges. Por el contrario, existe una clara sobrerrepresentación de latinoamericanos y asiáticos. En los municipios más alejados, en cambio, existe una mayor representación de africanos, que llegan a sumar más del $50 \%$ de los extranjeros presentes (es el caso de Mataró y Terrassa o de un número importante de municipios de tamaño medio). Los asiáticos muestran una pauta muy concentrada, representan el 19\% de los extranjeros en Barcelona, superando esta cifra en contadas ocasiones (Santa Coloma de Gramenet, 35,5\%; Badalona, 34,6\%; y Sant Adrià del Besòs, 20,6\%). En cambio, el porcentaje de americanos en Barcelona (42\%) es más elevado que en el conjunto de la RMB, y es muy importante en L'Hospitalet de Llobregat (63\%) o Sabadell (49,7\%). Finalmente, los europeos en Sant Just Desvern, Sitges o Sant Cugat del Vallès superan el $40 \%$ de los extranjeros presentes, por un $31,5 \%$ en Barcelona. En el resto de municipios metropolitanos, son mucho menos frecuentes.

En cuanto a la movilidad residencial centro-periferia, ésta actúa de forma selectiva en la ciudad de Barcelona. El resultado es un claro saldo migratorio neto negativo con el resto de la RMB (figura 2 y tabla 1). De esta manera, Barcelona, pero también los municipios más densos de su continuo urbano - primera corona - entran en una dinámica de pérdida continuada de población hacia sus periferias (tabla 1 y figura 2, arriba), decreciendo la población a todas las edades, en mayor medida entre los 25 y 34 años de edad.

TABLA 1

DINÁMICA DEMOGRÁFICA Y MIGRATORIA DE LOS MUNICIPIOS DE LA RMB SEGÚN DISTANCIA A LA CIUDAD CENTRAL, 1998-2009

\begin{tabular}{lccccc}
\hline & $\begin{array}{c}(1) \\
\text { Crecimiento } \\
\text { Natural }\end{array}$ & $\begin{array}{c}(2) \\
\text { Crecimiento } \\
\text { Total }\end{array}$ & $\begin{array}{c}\text { (3) }=(2)-(1) \\
\text { Migración } \\
\text { Neta }\end{array}$ & $\begin{array}{c}\text { (4) Saldo } \\
\text { Migratorio } \\
\text { interno (RMB) }\end{array}$ & $\begin{array}{c}(5)=(3) \text { - (4) Saldo } \\
\text { migratorio exterior } \\
\text { estimado a la RMB }\end{array}$ \\
\hline Barcelona & -25.734 & 122.201 & 147.935 & -186.611 & 334.546 \\
Menos de 10 km & 23.667 & 32.617 & 8.950 & -69.339 & 78.289 \\
Entre 10 y 20 km & 62.872 & 200.627 & 137.755 & 73.623 & 64.132 \\
Entre 20 y 30 km & 42.960 & 195.618 & 152.658 & 75.229 & 77.429 \\
Entre 30 y 40 km & 11.652 & 91.964 & 80.312 & 56.871 & 23.441 \\
Entre 40 y 50 km & 5.574 & 66.562 & 60.988 & 33.654 & 27.334 \\
Más de 50 km & 2.524 & 26.711 & 24.187 & 16.573 & 7.614 \\
RMB & 123.515 & 736.300 & 612.785 & 0 & 612.785 \\
\hline
\end{tabular}

Fuente: elaboración a partir de MNP, Estadística de Variaciones Residenciales (EVR) y Padrón Continuo de población, con datos del INE e Idescat. 
Desde la perspectiva de los hogares, existe una clara tendencia hacia el asentamiento preferente de las parejas jóvenes o parejas con hijos fuera de la ciudad central vía movilidad residencial (López-Villanueva et al., 2013)4. Al estar vinculada a la formación de una nueva pareja o con algún otro cambio en el curso de vida (como la llegada de un hijo), la movilidad residencial actúa, pues, de manera selectiva, redistribuyendo la población según determinadas características sociodemográficas y especializando, en consecuencia, el territorio. Las grandes ciudades se convierten en espacios envejecidos que sufren un rápido pero breve rejuvenecimiento por la llegada de extranjeros; al tiempo que las periferias se transforman paulatinamente en espacios de gran crecimiento, especializadas en poblaciones jóvenes y receptoras de familias con hijos. Esta estructura resultante ha influido, sin duda, en la evolución de la fecundidad de los diferentes municipios metropolitanos.

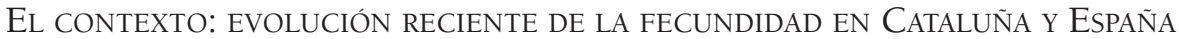

Actualmente, la fecundidad en España se sitúa en valores bajos en el contexto europeo, a pesar de haber experimentado recientemente un crecimiento significativo. En efecto, la fecundidad española era de las más elevadas de Europa en 1975 con casi 2,8 hijos por mujer (datos del INE), pero se redujo rápidamente durante la segunda mitad de los años setenta, la entera década de los ochenta y la primera mitad de los noventa hasta llegar a un mínimo de 1,15 hijos por mujer en 1998, que representaba uno de los valores más bajos del mundo en ese momento. Desde entonces inició una tendencia positiva empujada por la buena coyuntura económica, la necesidad de las mujeres españolas de las generaciones del baby-boom de completar su descendencia, y la llegada de numerosas mujeres extranjeras con pautas reproductivas, en general, más natalistas. Todo ello llevó la fecundidad española a aumentar progresivamente hasta un máximo de casi 1,46 hijos por mujer en 2008, aunque, y de forma reciente, la intensidad parece recuperar su camino descendente como consecuencia de la crisis económica, con un descenso hasta los 1,38 hijos por mujer observados en el año 2010.

\footnotetext{
${ }^{4}$ Por ejemplo, entre los hogares donde la persona de referencia realizó un cambio de municipio metropolitano entre 1991 y 2001, el 49,5\% de ellos eran formados por parejas en Barcelona, mientras que este porcentaje ascendía al $68,1 \%$ en los municipios de la segunda corona metropolitana.
} 
En el caso de Cataluña las tendencias son muy similares, con la particularidad de que la fecundidad media se situaba por debajo de la española desde 1976 (2,69 hijos por mujer, según el INE) hasta $1996(1,15)$, pero desde entonces se encuentra ligeramente por encima (1,58 en 2008 o 1,53 en 2010). Y esto tanto para las mujeres autóctonas (1,32 hijos por mujer en el total de España en 2010, para 1,38 en Cataluña) como para las extranjeras: 1,64 en el conjunto estatal por 1,94 en Cataluña, territorio en el que hay mayor presencia de las comunidades africanas y asiáticas, de mayor fecundidad relativa (Devolder y Bueno, 2011).

\section{La feCundidad en la Región Metropolitana de Barcelona (1986-2010)}

Además de una fecundidad situada durante las tres últimas décadas por debajo del nivel del reemplazo, el retraso en la edad media a la maternidad y el crecimiento del porcentaje de hijos de madre no casada son dos de las características más importantes observadas en la actualidad. En el caso específico de la Región Metropolitana de Barcelona la edad media a la maternidad sigue un curso ascendente hasta situarse, en 2010, en los 31,4 años, al tiempo que más de un tercio de los nacimientos se producen ya fuera de la institución matrimonial. Este es, quizás, el indicador con mayor variación durante las últimas décadas, ya que en 1986 apenas el 7,2\% de los nacimientos respondían a esta situación, y una clara expresión del cambio de valores que acompaña a la Segunda Transición Demográfica (Lesthaeghe, 1995 y Van de Kaa, 1987).

\section{La intensidad de la fecundidad}

Respecto a la intensidad de la fecundidad, los datos publicados por el Idescat desde 1986 para la RMB muestran unos ISF muy similares a los del conjunto de Cataluña, aunque algo inferiores, con valores de 1,37 hijos por mujer en 1986 frente a 1,39; de 1,13 en 1995 frente a 1,15; de 1,27 en 2000 frente a 1,28 ; de 1,47 en 2008 frente a 1,58; y de 1,40 en 2010 frente a 1,53 hijos por mujer, respectivamente.

Pero, como veremos a continuación, los datos de intensidad de la fecundidad referidos al conjunto de la región metropolitana ocultan grandes diferencias entre los municipios situados en su interior. En los años más recientes se ha producido una diferenciación creciente de comportamientos, que se ampli- 
TABLA 2

PRINCIPALES VARIABLES DE LA FECUNDIDAD METROPOLITANA, 1986-2010

\begin{tabular}{llrrrrrr}
\hline & & 1986 & 1991 & 1996 & 2001 & 2006 & 2010 \\
\hline \multirow{2}{*}{ Tasa bruta de } & Barcelona & 8,96 & 8,25 & 8,23 & 8,83 & 9,44 & 9,35 \\
natalidad (\%) & Restos RMB & 10,90 & 9,72 & 9,33 & 11,20 & 12,66 & 11,92 \\
& RMB & 10,12 & 9,15 & 8,94 & 10,40 & 11,60 & 11,09 \\
\hline Indicador & Barcelona & 1,27 & 1,13 & 1,13 & 1,18 & 1,19 & 1,18 \\
sintético & Restos RMB & 1,44 & 1,25 & 1,14 & 1,31 & 1,50 & 1,51 \\
fecundidad & RMB & 1,37 & 1,20 & 1,13 & 1,27 & 1,39 & 1,40 \\
\hline \multirow{2}{*}{ Edad media a } & Barcelona & 29,37 & 30,31 & 31,47 & 31,83 & 31,98 & 32,27 \\
la maternidad & Restos RMB & 28,33 & 29,07 & 30,26 & 30,83 & 30,90 & 31,11 \\
& RMB & 28,71 & 29,50 & 30,66 & 31,16 & 31,19 & 31,42 \\
\hline Nacimientos & Barcelona & 9,30 & 12,78 & 16,65 & 26,81 & 34,25 & 39,36 \\
de madre no & Restos RMB & 6,12 & 10,38 & 12,52 & 20,54 & 29,11 & 35,35 \\
casada \% & RMB & 7,25 & 11,21 & 13,88 & 22,36 & 30,49 & 36,44 \\
\hline
\end{tabular}

Fuente: MNP y Estimaciones de población intercensales y postcensales, con datos de Idescat.

fican con la recuperación de la fecundidad (tabla 2). La ciudad central anticipa el descenso de la fecundidad de los años ochenta y noventa, pero también el aumento de la edad media a la maternidad o de la proporción de nacimientos de madre no casada. En cambio, no participa de la recuperación de los niveles de fecundidad de los años más recientes, a pesar de contar con un número importante de extranjeros entre su población.

La evolución del índice sintético de fecundidad (ISF) entre los años 1986 y 2010 muestra, para los mayores municipios metropolitanos (figura 3), diferencias remarcables en su evolución e intensidad. Por un lado, la ciudad de Barcelona presenta valores caracterizados por mínimas variaciones en el tiempo, con unos máximos de 1,27 en 1986 o de 1,22 en 2008, y un mínimo de 1,10 en 1998. Tanto a inicios del periodo estudiado como en su final, el ISF estimado es el menor en comparación con el resto de ciudades de más de 100 mil habitantes de la RMB, situación que no se observa, en cambio, en la década de los noventa, cuando la fecundidad alcanzó sus valores mínimos. Durante parte de estos años, Santa Coloma de Gramenet y L'Hospitalet de Llobregat, dos ciudades situadas en la primera corona metropolitana y colindantes a la ciudad central, experimentan los índices más reducidos, con mínimos alrededor de 0,9 hijos por mujer a mitad de los años noventa. En cambio, ciudades más alejadas, situadas en la segunda corona metropolitana, como 
FIGURA 3

EVOLUCIÓN DEL ÍNDICE SINTÉTICO DE FECUNDIDAD (ISF)

EN LOS MUNICIPIOS MAYORES DE LA RMB, 1986-2010

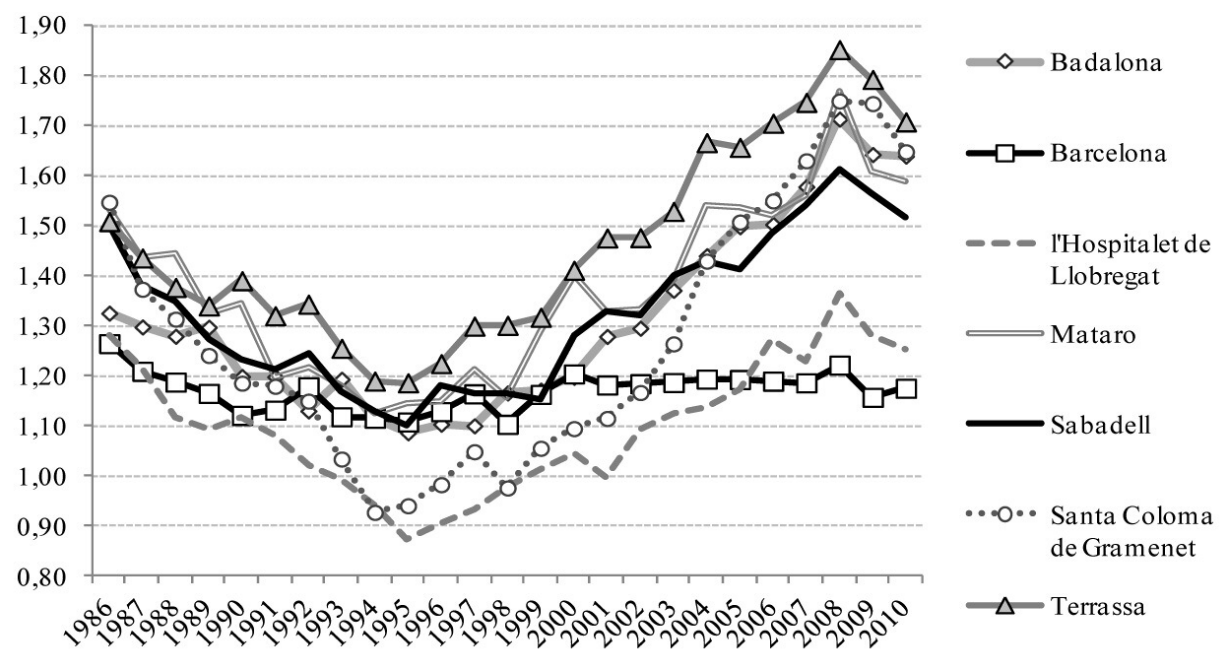

Fuente: elaboración propia a partir de MNP y estimaciones de población (Idescat).

Mataró y Terrassa, se mantienen durante estos años con los valores más elevados, con mínimos de 1,12 y 1,19 respectivamente en el año 1994, y máximos que alcanzan valores de 1,77 y de 1,85 en 2009. Es significativa la evolución de la fecundidad en Santa Coloma de Gramenet y LHospitalet de Llobregat durante los últimos años, ya que de presentar los valores más bajos a mediados de los noventa pasan, en muy poco tiempo, a superar la cifra de Barcelona e incluso, en el caso de Santa Coloma de Gramenet, a situarse entre las ciudades con mayor fecundidad (situación, por otro lado, que ya se producía en los años setenta y ochenta).

En el resto de la metrópoli, representada en los mapas de las figuras 4 y 5 , los mayores valores de fecundidad se observaban durante los primeros años analizados en las comarcas del Maresme y del Garraf (1,52 en 1991), máximos que a partir de 1996 se producen en Sant Cugat del Vallès (1,47 en 1996 o 1,67 en 2001), ciudad que experimenta un fuerte crecimiento de su población (de apenas 35 mil residentes en 1986 hasta superar los 83 mil en 2011), y que se sitúa entre los municipios con mayor renta disponible de la RMB. En los dos últimos años analizados la máxima fecundidad se da en Terrassa (1,71 en 2006 
FIGURA 4

EVOLUCIÓN DEL ÍNDICE SINTÉTICO DE FECUNDIDAD EN LA RMB, 1986-2010

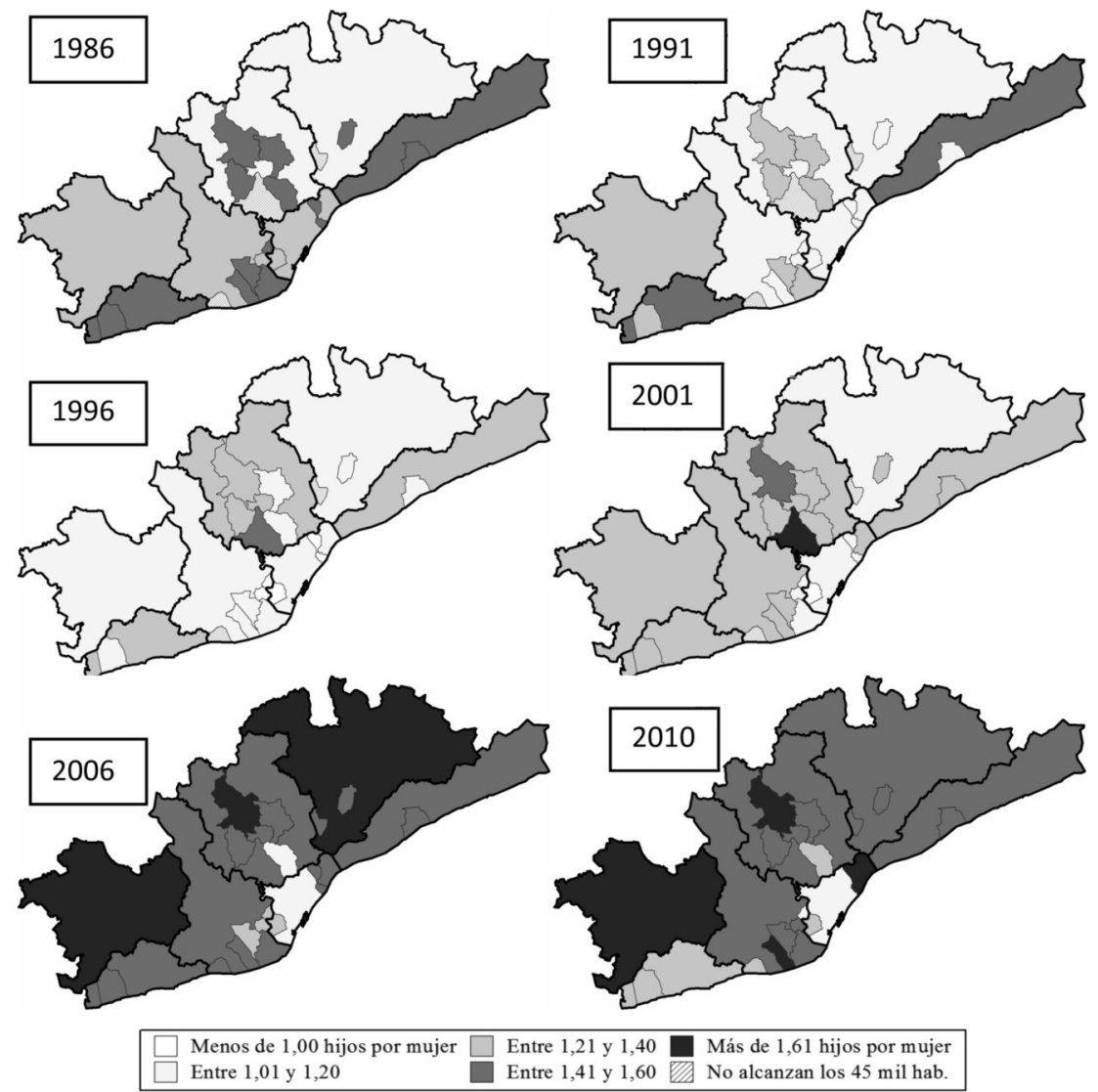

Fuente: elaboración propia a partir de MNP y estimaciones de población (Idescat).

y 2010), ciudad de la segunda corona con un fuerte crecimiento demográfico en los años más recientes (de 165 mil habitantes en 1998 a 212 mil en 2010).

Durante las primeras fechas analizadas los mínimos se encuentran en los restos comarcales del Vallès Oriental y Occidental (1,05 y 1,03 en 1991, respectivamente), mientras que en la década de los noventa se desplazan a L'Hospitalet de Llobregat $(0,91$ en 1996) y a otros municipios de la primera corona metropolitana como Santa Coloma de Gramenet, Esplugues o Cornellà. En el presente siglo, en cambio, es Cerdanyola del Vallès la que tiene la menor fe- 
FIGURA 5

EVOLUCIÓN DEL ÍNDICE SINTÉTICO DE FECUNDIDAD (ISF)

EN LOS MUNICIPIOS DE TAMAÑO MEDIO DE LA RMB, 1986-2010

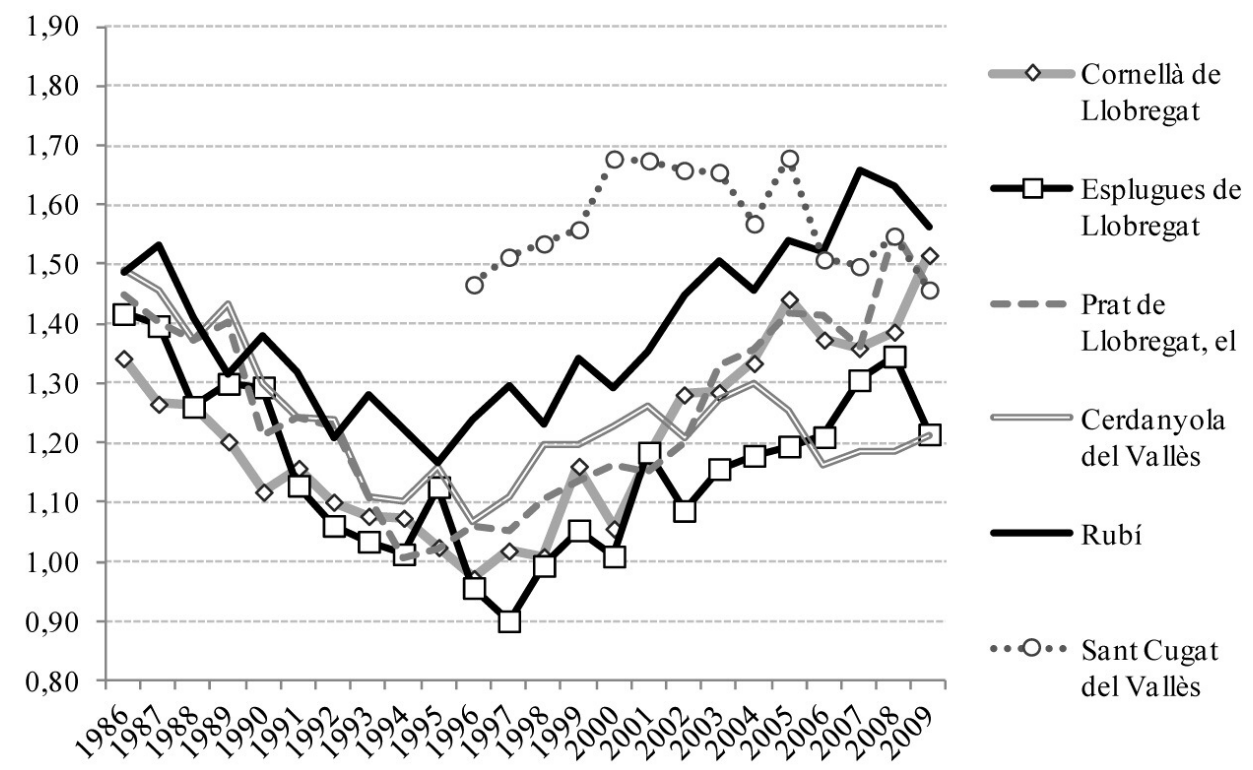

Fuente: elaboración propia a partir de MNP y estimaciones de población (Idescat).

cundidad (1,16 hijos por mujer en 2006 y 1,21 en 2010) junto con la ciudad de Barcelona, que ha ido quedando progresivamente rezagada en las posiciones inferiores del ranking.

Esta evolución tan poco dinámica de la fecundidad en la capital catalana se observa perfectamente en la figura 6 (izquierda), que resume la evolución de los valores máximos y mínimos observados en la RMB en relación con Barcelona (el gráfico de la derecha muestra la misma evolución en relación con la media de la RMB). Se ve perfectamente que la recuperación de la fecundidad iniciada a finales de los años noventa no tiene lugar en la ciudad de Barcelona, acrecentándose por lo tanto las diferencias entre centro y periferias metropolitanas. Esta segmentación de comportamientos entre ambos ámbitos puede deberse, a falta de un análisis de mayor profundidad, a la salida de la ciudad central de las parejas jóvenes que desean tener hijos, con independencia de su nacionalidad. 
FIGURA 6

VALORES MÁXIMOS Y MÍNIMOS DEL ÍNDICE SINTÉTICO DE FECUNDIDAD (ISF) EN RELACIÓN A BARCELONA (IZQ.) Y A LA MEDIA DE LA RMB (DER.), 1986-2010
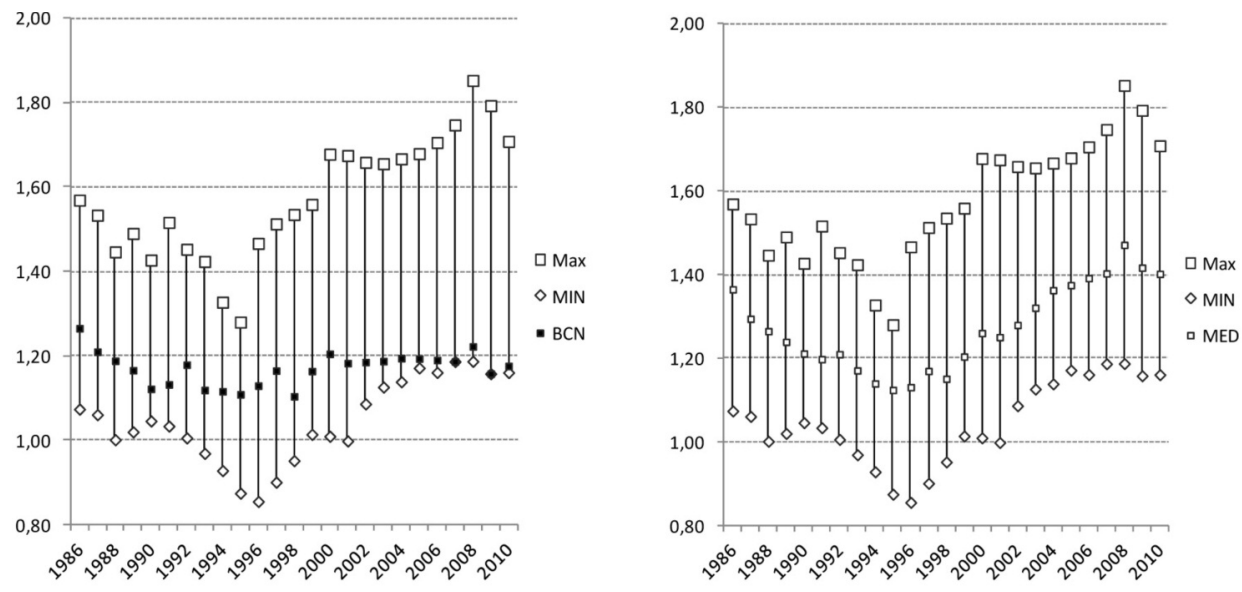

Fuente: elaboración propia a partir de MNP (Idescat) y estimaciones de población (Idescat).

\section{La estructura por edades de la fecundidad}

Por último, abordamos la evolución del calendario de la fecundidad tanto en Barcelona como en una serie de municipios metropolitanos representati$\operatorname{vos}^{5}$ (figura 7 y figura 8). El retraso a la fecundidad es evidente en la ciudad de Barcelona, donde las tasas máximas se desplazan de los 28 a los 34 años entre 1986 y 2009, a pesar de un ligero incremento de la maternidad en las edades más jóvenes que se observa desde 2001, y que sería atribuible a la fecundidad de las mujeres extranjeras, las cuales presentan un calendario de la fecundidad mucho más joven. Este último fenómeno se produce con mayor intensidad en los otros tres casos seleccionados, en especial en Santa Coloma de Gramenet, municipio con fuerte presencia de mujeres procedentes de países africanos y asiáticos. De hecho, si analizamos la evolución de la fecundidad en los cuatro municipios seleccionados se observa cómo el incremento de la in-

\footnotetext{
${ }^{5}$ L'Hospitalet de Llobregat, Santa Coloma de Gramenet y Terrassa.
} 


\section{FIGURA 7}

TASAS DE FECUNDIDAD GENERAL POR EDAD (1986, 1995, 2001 Y 2009)

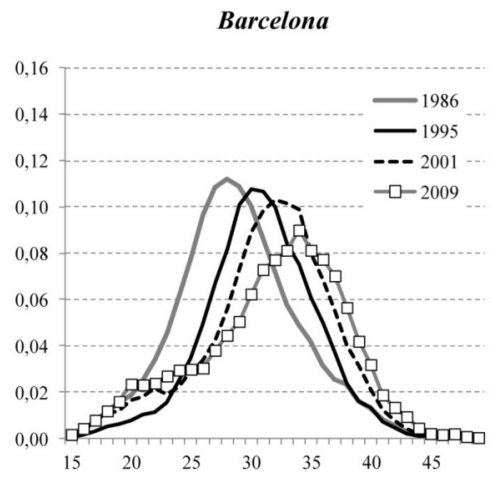

Santa Coloma de Gramenet

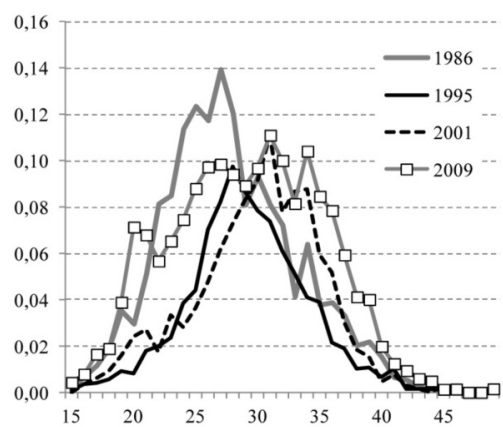

L'Hospitalet de Llobregat

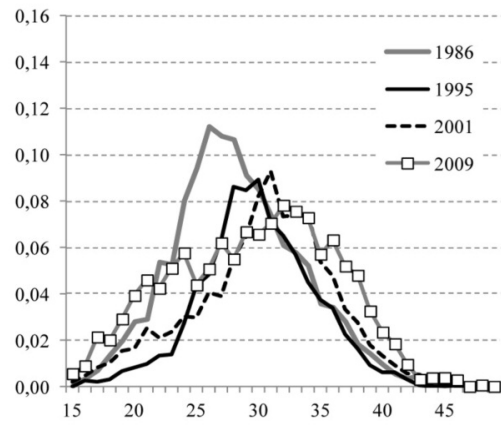

Terrassa

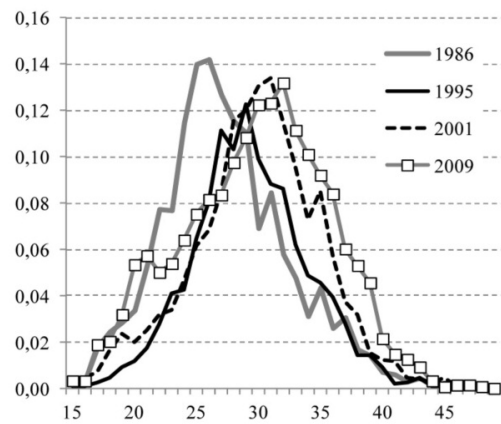

Fuente: elaboración propia a partir de MNP (Idescat) y estimaciones de población (Idescat).

tensidad observado entre 2001 y 2009 no es atribuible a una mayor intensidad fecunda en las edades centrales sino a un aumento de las tasas tanto antes de los 25 años (atribuible sobre todo a las mujeres extranjeras) como después de los 35 (causado sobre todo por el retraso de las mujeres españolas).

Como resultado, en Barcelona la edad media a la maternidad en 2009 ya supera los 32 años (32,2 exactamente), y se diferencia del resto metropolitano, que tiene en conjunto un calendario más joven $(30,9)$. Por municipios, el calendario tan tardío de Barcelona tan sólo se supera en Sant Cugat del Vallès (33,1 años), aunque en este caso el ISF es más elevado $(1,46)$ debido al establecimiento de parejas adultas de nacionalidad mayoritariamente española 
FIGURA 8

TASAS DE FECUNDIDAD GENERAL POR EDAD, 1986 Y 2009. CIUDADES SELECCIONADAS

1986

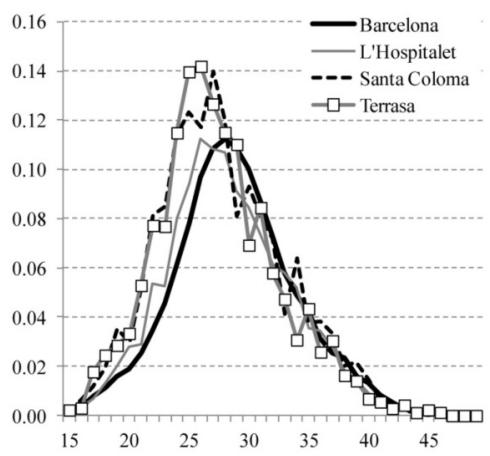

2009

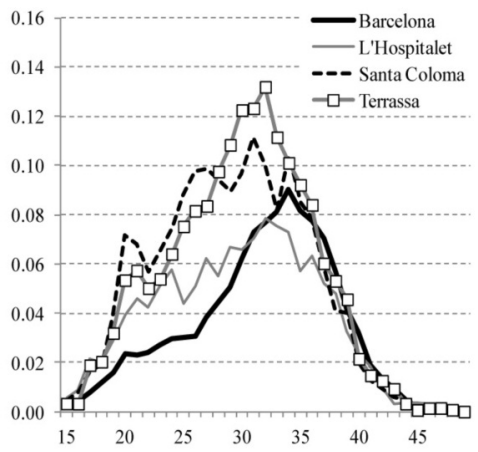

Fuente: elaboración propia a partir de MNP (Idescat) y estimaciones de población (Idescat).

que emigran a ese municipio residencial de clase media-alta con el objetivo de formar una familia o ampliarla. Por el contrario, la edad media a la maternidad más baja se produce en Santa Coloma de Gramenet, por debajo de los 30 años $(29,6)$, siendo en este caso municipio de recepción de parejas jóvenes con un fuerte componente extranjero, como se demostrará a continuación.

\section{Los nacimientos de madre extranjera}

Uno de los elementos que aparecen como determinantes en los niveles actuales de fecundidad, y particularmente de las diferencias existentes entre municipios, es la participación de la población extranjera en dicho fenómeno. En 2010, de los 486.575 nacimientos registrados en España, un 24,1\% de ellos (117.238) eran hijos de al menos uno de los padres con nacionalidad extranjera; en Cataluña este porcentaje es significativamente más elevado, ya que 28.435 nacimientos, el 33,7\% del total, responden a esta categoría. De estos últimos, un $45,3 \%$ era hijo de un padre/madre español y un extranjero. A continuación se analiza la participación de los niños de madres extranjeras sobre el total de nacimientos en la RMB para el periodo 2000-2010, utilizando datos del Idescat por lugar de residencia de la madre. 
En el año 2000, la proporción de nacimientos de madre extranjera era de un $8,2 \%$ de los nacimientos producidos en la RMB, 3.665 sobre un total de 44.438. A finales de la década, mientras que los nacimientos de madre española habían incluso descendido ligeramente (de 40.773 en 2000 a 40.333 de 2010), los de madre extranjera se habían cuadruplicado, alcanzando los 14.753, que representan más de uno de cada cuatro nacimientos en la RMB (el 26,8\%), que por aquel entonces habían ascendido a 55.086. Este porcentaje tan significativo es incluso superior en el conjunto de Cataluña, donde un $29 \%$ de los nacimientos (24.391 sobre 84.015) eran, en 2010, hijos de madre extranjera.

A nivel comarcal dentro de la Región metropolitana, en el año 2000 los porcentajes oscilaban entre un mínimo del $5,1 \%$ en el Vallès Occidental a un máximo del 10,8\% en el Maresme, valores que en 2010 son del 21,4\% en el Baix Llobregat como mínimo al máximo del 33,1\% en el Barcelonès. A nivel municipal, las disparidades son incluso mucho más acusadas, y ello puede ayudar a explicar, siquiera parcialmente, las diferencias de intensidad de fecundidad existentes: si en 2000 algunos de estos porcentajes apenas se situaban en un bajo 3,3\% (Viladecans, a pesar de ser un municipio con una larga tradición de inmigración magrebí), con un máximo del 13,6\% en Mataró (con un fuerte componente subsahariano entre los inmigrantes); en 2010 estos umbrales se sitúan entre el mínimo del 13,5\% en Viladecans a un máximo del 46,7\% de LHospitalet de Llobregat, ciudad con una inmigración básicamente latinoamericana. Por su parte, la proporción de nacimientos de madre extranjera asciende al 30,8\% en la ciudad de Barcelona, mientras que en Santa Coloma de Gramenet alcanza el 45,9\%, es decir, muy similar al máximo de L'Hospitalet y cercano a casi la mitad de los nacimientos. Esta es una línea de investigación que estamos siguiendo actualmente y en la que profundizaremos en próximos trabajos, pero aunque sea a nivel descriptivo podemos observar la existencia de una relación, en ciertos municipios, entre el porcentaje de nacimientos de madre extranjera, el calendario más joven de su fecundidad y el incremento de su intensidad en los últimos años.

\section{CONCLUSIONES}

Los resultados de esta investigación han verificado la hipótesis de partida, demostrando que las diferencias de fecundidad son progresivamente más acusadas entre los distintos ámbitos de la RMB. Ello sería un reflejo de la creciente especialización productiva y residencial del territorio metropolitano, que a su vez se traduce en preferencias de asentamiento según la nacionalidad 
y la fase del ciclo de vida. Las consecuencias directas de estos comportamientos se reflejan también en la estructura por edades, con un centro progresivamente más envejecido a pesar del paréntesis que la incorporación de extranjeros significa sobre la evolución de este indicador, y de unas periferias rejuvenecidas gracias a la llegada de parejas en fase de creación o ampliación de su descendencia.

Esta pauta de creciente diferenciación entre el centro y sus periferias se reflejaría, en primer lugar, en la ciudad de Barcelona, que muestra una fecundidad baja y muy estable en el tiempo durante los últimos 25 años, con niveles prácticamente inamovibles a pesar de la tendencia general de ascenso o descenso. Por un lado no experimenta el brusco descenso de las ciudades colindantes que se observó entre mediados de los años setenta y mediados de los noventa, cuando apenas superaban el valor de 0,9 hijos por mujer, mientras que durante los últimos años Barcelona tampoco participa del crecimiento de la fecundidad y se sitúa gradualmente entre los valores más bajos de la RMB. El retraso de la fecundidad que se observa en la ciudad central (con la edad media a la maternidad más elevada), no ayuda a la convergencia con los valores del resto metropolitano, situándose el ISF de Barcelona cada vez más lejos de la media de la región. Por otro lado la elevada edad media a la fecundidad hace pensar en la existencia de una elevada infecundidad (población femenina sin hijos) en la población de Barcelona.

En segundo lugar, los municipios de la periferia inmediata de Barcelona, esto es, los municipios del continuo urbano o primera corona metropolitana, con alto impacto de la inmigración extranjera, tienen un comportamiento peculiar al pasar, en pocos años, de mostrar los niveles de fecundidad más bajos de la RMB a mediados de los noventa, a otros por encima de la media en los años más recientes. Ello se debe a que, a pesar de que también pierden población española en dirección a la periferia más exterior, reciben al mismo tiempo a muchos inmigrantes de nacionalidad extranjera procedentes de la ciudad central o del exterior, y de edad mayoritariamente joven, que se asientan en dichos municipios (bien comunicados con Barcelona por transporte público y con abundantes viviendas de calidad media-baja a precios más asequibles) para iniciar o desarrollar su proyecto reproductivo.

Por último, los municipios de las coronas más alejadas se caracterizan por una fecundidad elevada y creciente, al menos hasta el inicio de la crisis. Ello se debe a que reciben una mayor inmigración relativa por movilidad residencial de individuos y familias, mayoritariamente jóvenes y de nacionalidad española ( $y$, crecientemente, también de origen extranjero), procedentes de Barcelona y los municipios de su continuo urbano. En estas áreas sí que en- 
contramos ciudades que mantienen niveles de fecundidad por encima de la media (Mataró o Terrassa), situación que debe relacionarse con un incremento significativo del parque de viviendas de estas urbes.

Del análisis de los datos presentados se desprenden algunas ideas que deberán ser analizadas con mayor detalle en nuevas aproximaciones. Por un lado, apuntamos que no existe una relación directa entre mayores niveles de fecundidad y mayor porcentaje de población extranjera. Esto es así al menos en el caso de Barcelona, que durante los años de mayores entradas de inmigrantes se mantuvo entre los municipios de la RMB con el porcentaje de extranjeros más elevado, pero no con los mayores niveles de fecundidad. En cambio, la presencia de extranjeros sí que incide directamente en el incremento de la fecundidad de los municipios de la primera corona metropolitana, siendo los casos de L'Hospitalet de Llobregat y más claramente el de Santa Coloma de Gramenet los más relevantes. Ello se podría interpretar como que los extranjeros, de forma similar a los españoles, no considerarían la gran ciudad central (Barcelona) como el lugar más adecuado para llevar a cabo su proyecto familiar, quizás por la escasez de viviendas con las características que consideran adecuadas (pisos de varias habitaciones con un precio asequible para sus mayoritariamente modestas economías). La ciudad central es asimismo un primer punto de entrada de extranjeros que luego consolidan su proyecto vital, y también familiar, en otro municipio metropolitano. Al mismo tiempo, las diferencias de intensidad respecto a la fecundidad encontradas entre las dos ciudades citadas, que se podrían relacionar con las nacionalidades presentes en uno $\mathrm{u}$ otro municipio - mayor presencia de latinoamericanos en L'Hospitalet, de africanos y asiáticos en Santa Coloma-, demuestran que es necesario un análisis más detallado de la fecundidad de la población extranjera a nivel municipal. De la misma manera, la coincidencia en la segunda corona metropolitana de los flujos migratorios suburbanos protagonizados por españoles y la presencia de población africana, con mayor fecundidad, inciden en esa necesidad de discernir el papel de ambos componentes en el crecimiento de la fecundidad, línea de investigación que se tendrá que seguir a partir de los datos del Padrón Continuo.

Otro aspecto a considerar en el futuro es la relación de la fecundidad con la estructura social del territorio, debido a la posible existencia de pautas reproductivas que difieran por nivel socio-económico. Además, si la fecundidad se encuentra ligada estrechamente a la coyuntura económica, será a nivel municipal donde los efectos de la crisis se observarán más intensamente, en función de los rasgos socio-económicos de cada municipio. Dicho estudio se podrá realizar cuando contemos con los datos completos del Censo de 2011. 
En último lugar, queda por analizar la magnitud del impacto directo de la movilidad residencial, ya que el análisis exploratorio parece mostrar que unos saldos migratorios más positivos parecen ir correlacionados con una mayor fecundidad de dichos municipios receptores. Esto se debería al protagonismo de las parejas jóvenes en este fenómeno, sin despreciar un posible comportamiento diferencial respecto a la fecundidad en función del lugar de residencia - es decir, sin efectuar un cambio municipal previo-, que también debería analizarse. El análisis de los factores que presuntamente afectan a los niveles municipales de fecundidad (sean el porcentaje de población extranjera, la magnitud de los saldos migratorios, o las características socio-demográficas de la población asentada en dichos municipios, entre otros) merecen una investigación más profunda que estamos llevando a cabo actualmente sobre la base de los resultados descriptivos presentados en este artículo.

Fecha de recepción: 15/10/2012

Fecha de aceptación: 01/07/2013

\section{BIBLIOGRAFÍA}

Alberich, J. (2010): "Lús de l'espai". Papers de la Regió Metropolitana de Barcelona, 51 (Transformacions territorials a l'Àrea Metropolitana de Barcelona. Una visió a partir de l'ECVHP), pp. 28-43.

Bayona, J. y Gil-Alonso, F. (2012): "Suburbanisation and international immigration: The case of the Barcelona Metropolitan Region (1998-2009)". Tijdschrift voor economische en sociale geografie, 103/3, pp. 312-329.

Bayona, J., Gil-Alonso, F. y Pujadas, I. (2011): "Dinàmica residencial de la població estrangera en les principals regions metropolitanes d'Espanya". Revista Catalana de Sociologia, 27, pp. 15-32.

Bayona, J. y Pujadas, I. (2014): "Movilidad residencial y redistribución de la población metropolitana: los casos de Madrid y Barcelona" EURE, 119.

Boyle, P. (2003): "Population geography: does geography matter in fertility research?". Progress in Human Geography, 27/5, pp. 615-626.

Buzar, S., Odgen, P. E. y Hall, R. (2005): "Households matter: the quiet demography of urban transformation". Progress in Human Geography, 29/4, pp. 413-436.

Cabré, A. y Módenes, J. A. (1997): "Dinàmiques demogràfiques a la Regió Metropolitana de Barcelona". Revista Econòmica de Catalunya, 33, pp. 66-76.

Castro, T. y Rosero-Bixby, L. (2011): "Maternidades y fronteras: La fecundidad de las mujeres inmigrantes en España". Revista Internacional de Sociología, 1, pp. 105-137.

Courgeau, D. (1989): "Family Formation and Urbanisation". Population: an English Selection, 44/1, pp. 123-146. 
Devolder, D., Bueno, X., Gumá, J., Treviño, R. y Zueras, P. (2010): Anàlisis de la fecunditat a partir de l'Enquesta demogràfica de Catalunya, 2007, Col. Quaderns d'Estadística,4, desembre 2010. Barcelona, Generalitat de Catalunya, Institut d'Estadística de Catalunya (IDESCAT), 193 pp.

Devolder, D. y Bueno, X. (2011): "Interacciones entre fecundidad y migración. Un estudio de las personas nacidas en el extranjero y residentes en Cataluña en 2007". Documents d'Anàlisi Geogràfica, 57/3, pp. 441-467.

Devolder, D. y Cabré, A. (2009): "Factores de la evolución de la fecundidad en España en los últimos 30 años". Panorama Social, 10, (Familias en Transformación) pp. 23-39.

Domingo, A. y Bayona, J. (2007): "Migracions, població estrangera i habitatge a la ciutat de Barcelona en el context metropolità", en A. Costas (dir.): Llibre blanc de l'habitatge a Barcelona. Barcelona, Ajuntament de Barcelona, pp. 16-60. Disponible: http://www.bcn.es/conselldeciutat/pdf/lb_PART_II_03_Poblacio_habitatge_ Barcelona_context.pdf (Fecha de consulta: 26/07/2013).

Feria, J. M. y Susino, J. (2012): "Movilidad residencial y configuración metropolitana en España", en P. Reques y O. De Cos (ed.): La población en clave territorial. Procesos, estructuras y perspectivas de análisis. Santander, Asociación de Geógrafos Españoles y Universidad de Cantabria, pp. 60-70.

Gil-Alonso, F. (2010): "Análisis de dos propuestas metodológicas para estimar las salidas de extranjeros de España: las bajas por caducidad padronales y la renovación de las tarjetas de residencia temporales". Estadística Española, 52/174, pp. 277-309.

Kulu, H. y Boyle, P. (2009): "High Fertility in City Suburbs: Compositional or Contextual Effects?". European Journal of Population, 25, pp. 157-174.

Kulu, H., Vikat, A. y Andersson, G. (2007): "Settlement size and fertility in the Nordic countries". Population Studies, 61/3, pp. 265-285.

Kulu, H., Boyle, P. y Andersson, G. (2009): "High suburban fertility: Evidence from four Northern European countries". Demographic Research, 21/31, pp. 915-944.

Laslett, P. (1972): "Introduction: The History of the family", en P. Laslett (ed.): Household and Family in Past Time. Cambridge, Cambridge University Press, pp. 1-86.

Lesthaeghe, R. (1995): "The Second Demographic Transition in Western Countries: An Interpretation", en K. Mason y A-M. Jensen (ed.): Gender and Family Change in Industrialized Countries. Oxford, Clarendon Press, pp. 17-62.

López-Villanueva, C., Pujadas, I. y Bayona, J. (2013): "Households Within The Residential Mobility Process: The Case Of The Barcelona Metropolitan Region" Archivio di Studi Urbani e Regionali, 108.

Michielin, F. (2004): "Lowest Low Fertility in an Urban Context: The Role of Migration in Turin, Italy". Population, Space and Place, 10, pp. 331-347.

Módenes, J. A. (1998): "Flujos espaciales e itinerarios biográficos: la movilidad residencial en el área de Barcelona”. Tesis Doctoral. Departamento de Geografía, UAB, Barcelona.

Pozo, E. y García Palomares, J. C. (2011): "Evolución reciente y pautas de distribución espacial de las migraciones internas de extranjeros: el caso de la Comunidad 
de Madrid (1997-2008)". Scripta Nova. Revista Electrónica de Geografía y Ciencias Sociales, XV /384.

Pozo, E. y Rodríguez Moya, J. M. (2003): "Diferencias socioespaciales en la fecundidad madrileña en el cambio de siglo”. Estudios Geográficos, LXIV/252, pp. 455-486.

Pozo, E. y Rodríguez Moya, J. M. (2002): "Cambios recientes de la fecundidad en la Comunidad de Madrid". Anales de Geografía de la Universidad Complutense, Vol. Extraordinario 2002, pp. 399-408.

Pujadas, I. (2009): "Movilidad residencial y expansión urbana en la Región Metropolitana de Barcelona, 1982-2005". Scripta Nova. Revista Electrónica de Geografía y Ciencias Sociales, XIII/290.

Pujadas, I. y López-Villanueva (2005): "Hogares y cambios residenciales: la diferenciación espacial de los hogares en la Región Metropolitana de Barcelona, 19862001". Cuadernos Geográficos, 36, pp. 409-435.

Pujadas, I., Bayona, J. y Gil-Alonso, F. (2012): "Las grandes metrópolis españolas en la encrucijada. Crecimiento, migración y suburbanización en la última década". Contexto, Revista de la Facultad de Arquitectura Universidad Autónoma de Nuevo León, 6, pp. 11-32.

Pujadas, I. (1992): "La fecunditat" en Institut d'Estudis Metropolitans de Barcelona (IEMB) (ed.): Enquesta de la Regió Metropolitana de Barcelona 1990. Condicions de vida i hàbits de la población. 1. Aspectes demogràfics i característiquesfamiliars i relacionals. Barcelona, MMAMB, pp. 51-69.

Reques, P. (2011): "Transformaciones espaciales y procesos sociodemográficos en la ciudad", en Pujadas et al. (eds.): Población y Espacios Urbanos. Barcelona, Asociación de Geógrafos Españoles y Departamento de Geografía Humana de la Universidad de Barcelona, pp. 235-284.

Van de Kaa, D. (1987): "Europe's Second Demographic Transition". Population Bulletin. 42/1, pp. 1-59.

\section{RESUMEN}

Este trabajo analiza las diferencias en los valores de fecundidad dentro de una misma área metropolitana, partiendo de la hipótesis de que los comportamientos demográficos se encuentran cada vez más segmentados territorialmente en el interior de las metrópolis debido a la actuación de dos dinámicas que han alcanzado recientemente una gran intensidad: la masiva llegada y asentamiento de inmigrantes extranjeros y la redistribución de la población — sea autóctona o extranjera- dentro de la propia área urbana, proceso que se conoce como suburbanización. Se toma la Región Metropolitana de Barcelona (RMB) como caso de estudio, y se calcula el Índice Sintético de Fecundidad (ISF) para 26 subáreas metropolitanas (19 municipios mayores de 45 mil habitantes y 7 restos comarcales). Los resultados confirman la hipótesis y muestran la creciente diferenciación de los comportamientos demográficos, y concretamente de la fecundidad: la ciudad de Barcelona aparece con una fecundidad mucho más baja que 
la de los municipios suburbanos de la segunda corona metropolitana, mientras que los grandes municipios de la primera corona son los que más han aumentado su intensidad en los últimos años, partiendo de niveles muy bajos, como consecuencia del asentamiento de mujeres inmigrantes, aunque con diferencias sensibles de fecundidad en función de la nacionalidad de éstas.

Palabras Clave: fecundidad; área metropolitana; comportamientos diferenciales; análisis espacial de la población; Barcelona.

\section{Abstract}

The paper's main aim is to analyse fertility differences within a metropolitan area. Our initial hypothesis is that, due to two recent and intense processes, international migration and suburbanisation, intra-metropolitan demographic behaviour is increasingly spatially segmented. To this aim we have used the Metropolitan Region of Barcelona (RMB) as a study case and have calculated the Total Fertility Rate (TFR) for 26 metropolitan subareas. Results confirm our hypothesis as the core city has lower fertility rates than the rest of metropolitan municipalities, while those belonging to the suburban inner-ring, where a large amount of immigrant women have settled, currently have the highest rates.

KEY WorDs: fertility; metropolitan area; differential demographic behaviours; population spatial analysis; Barcelona.

\section{RÉSUMÉ}

Cet article analyse les différences de fécondité à l'intérieur d'une même région métropolitaine. Notre hypothèse initiale est que ces différences sont de plus en plus fortes à cause de l'influence de deux processus récents et très intenses : l'arrivée et l'établissement en masse d'immigrants étrangers et la redistribution de la population, qu'elle soit autochtone ou étrangère, à l'intérieur de l'aire urbaine en question, processus dénommé suburbanisation. Nous avons choisi comme cas d'étude la région métropolitaine de Barcelone (RMB). Plus précisément, nous avons calculé l'indicateur conjoncturel de fécondité pour 26 sous-régions de la RMB. Les résultats confirment notre hypothèse initiale et prouvent la différentiation croissante des comportements démographiques, et particulièrement de la fécondité. La ville de Barcelone présente un taux de fécondité beaucoup plus réduit que celui des communes suburbaines de la deuxième couronne métropolitaine, tandis que les grandes communes de la première couronne sont celles qui ont augmenté le plus leur intensité ces dernières années, en partant de niveaux très bas, à la suite de l'établissement de femmes immigrées, mais avec des différences sensibles de fécondité selon leur nationalité.

MotS CLÉS : fécondité; région métropolitaine; différences dans les comportements démographiques; analyse spatiale de la population; Barcelone. 